\title{
Software Infrastructure for Natural Language Processing
}

\author{
Hamish Cunningham \\ Dept. Computer Science \\ University of Sheffield \\ 211 Portobello St. \\ Sheffield S10 4DP \\ hamish@dcs . shef . ac .uk
}

\author{
Kevin Humphreys \\ Dept. Computer Science \\ University of Sheffield \\ 211 Portobello St. \\ Sheffield S10 4DP \\ kwh@dcs.shef.ac.uk
}

\author{
Robert Gaizauskas \\ Dept. Computer Science \\ University of Sheffield \\ 211 Portobello St. \\ Sheffield S10 4DP \\ robertg@dcs .shef .ac.uk
}

\author{
Yorick Wilks \\ Dept. Computer Science \\ University of Sheffield \\ 211 Portobello St. \\ Sheffield S10 4DP \\ yorick@dcs.shef .ac.uk
}

\begin{abstract}
We classify and review current approaches to software infrastructure for research, development and delivery of NLP systems. The task is motivated by a discussion of current trends in the field of NLP and Language Engineering. We describe a system called GATE (a General Architecture for Text Engineering) that provides a software infrastructure on top of which heterogeneous NLP processing modules may be evaluated and refined individually, or may be combined into larger application systems. GATE aims to support both researchers and developers working on component technologies (e.g. parsing, tagging, morphological analysis) and those working on developing end-user applications (e.g. information extraction, text summarisation, document generation, machine translation, and second language learning). GATE promotes reuse of component technology, permits specialisation and collaboration in large-scale projects, and allows for the comparison and evaluation of alternative technologies. The first release of GATE is now available.
\end{abstract}

\section{Introduction}

This paper reviews the currently available design strategies for software infrastructure for NLP and presents an implementation of a system called GATE - a General Architecture for Text Engineering. By software infrastructure we mean what has been variously referred to in the literature as: software architecture; software support tools; language engineering platforms; development environments. Our gloss on these terms is: common models for the representation, storage and exchange of data in and between processing modules in NLP systems, along with graphical interface tools for the management of data and processing and the visualisation of data. NLP systems produce information about texts ${ }^{1}$, and existing systems that aim to provide software infrastructure for NLP can be classified as belonging to one of three types according to the way in which they treat this information:

additive, or markup-based:

information produced is added to the text in the form of markup, e.g. in SGML (Thompson and McKelvie, 1996);

referential, or annotation-based: information is stored separately with references back to the original text, e.g. in the TIPSTER architecture (Grishman, 1996);

abstraction-based: the original text is preserved in processing only as parts of an integrated data structure that represents information about the text in a uniform theoretically-motivated model, e.g. attribute-value structures in the ALEP system (Simkins, 1994).

A fourth category might be added to cater for those systems that provide communication and control infrastructure without addressing the text-specific needs of NLP (e.g. Verbmobil's ICE architecture (Amtrup, 1995)).

We begin by reviewing examples of the three approaches we sketched above (and a system that falls into the fourth category). Next we discuss current trends in the field and motivate a set of requirements that have formed the design brief for GATE, which is then described. The initial distribution of the system includes a MUC-6 (Message Understanding Conference 6 (Grishman and Sundheim, 1996)) style information extraction (IE) system and an overview

\footnotetext{
${ }^{1}$ These texts may sometimes be the results of automatic speech recognition - see section 2.6.
} 
of these modules is given. GATE is now available for research purposes - see

http://wrw.dcs.shef.ac.uk/research/groups/ nlp/gate/ for details of how to obtain the system. It is written in $\mathrm{C}++$ and $\mathrm{Tcl} / \mathrm{Tk}$ and currently runs on UNIX (SunOS, Solaris, Irix, Linux and AIX are known to work); a Windows NT version is in preparation.

\section{Managing Information about Text}

\subsection{Abstraction Approaches}

The abstraction-based approach to managing information about texts is primarily motivated by theories of the nature of the information to be represented. One such position is that declarative, constraint-based representations using featurestructure matrices manipulated under unification are an appropriate vehicle by which "many technical problems in language description and computer manipulation of language can be solved" (Shieber, 1992). Information in these models may be characterised as abstract in our present context as there is no requirement to tie data elements back to the original text - these models represent abstractions from the text.

One recent example of an infrastructure project based on abstraction is ALEP - the Advanced Language Engineering Platform (Simkins, 1994). ALEP aims to provide "the NLP research and engineering community in Europe with an open, versatile, and general-purpose development environment". ALEP, while in principle open, is primarily an advanced system for developing and manipulating feature structure knowledge-bases under unification. Also provided are several parsing algorithms, algorithms for transfer, synthesis and generation (Schütz, 1994). As such, it is a system for developing particular types of data resource (e.g. grammars, lexicons) and for doing a particular set of tasks in LE in a particular way. ALEP does not aim for complete genericity (or it would need also to supply algorithms for Baum-Welch estimation, fast regular expression matching, etc.). Supplying a generic system to do every LE task is clearly impossible, and prone to instant obsolescence in a rapidly changing field.

In our view ALEP, despite claiming to use a theory-neutral formalism (an HPSG-like formalism), is still too committed to a particular approach to linguistic analysis and representation. It is clearly of high utility to those in the LE community to whom these theories and formalisms are relevant; but it excludes, or at least does not actively support, all those who are not, including an increasing number of researchers committed to statistical, corpus-based approaches. GATE, as will be seen below, is more like a shell, a backplane into which the whole spectrum of LE modules and databases can be plugged. Components used within GATE will typically exist already - our emphasis is reuse, not reimplementation. Our project is to provide a flexible and efficient way to combine LE components to make LE systems (whether experimental or for delivered applications) - not to provide 'the one true system', or even 'the one true development environment'. Indeed, ALEPbased systems might well provide components operating within GATE. Seen this way, the ALEP enterprise is orthogonal to ours - there is no significant overlap or conflict.

In our view the level at which we can assume commonality of information, or of representation of information, between LE modules is very low, if we are to build an environment which is broad enough to support the full range of LE tools and accept that we cannot impose standards on a research community in flux. What does seem to be a highest common denominator is this: modules that process text, or process the output of other modules that process text, produce further information about the text or portions of it. For example, part-of-speech tags, phrase structure trees, logical forms, discourse models can all be seen in this light. It would seem, therefore, that we are on safe common ground if we start only by committing to provide a mechanism which manages arbitrary information about text.

There are two methods by which this may be done. First, one may embed the information in the text at the relevant points - the additive approach. Second, one may associate the information with the text by building a separate database which stores this information and relates it to the text using pointers into the text - the referential approach. The next two subsections discuss systems that have adopted these two approaches respectively, then we compare the two and indicate why we have chosen a hybrid approached based mainly on the second. Finally we look at a system that falls outside our three categories.

\subsection{Additive Approaches}

Additive architectures for managing information about text add markup to the original text at each successive phase of processing. This model has been adopted by a number of projects including parts of the MULTEXT EC project. The MULTEXT work $^{2}$ has led to the development of an architecture based

\footnotetext{
${ }^{2}$ Note that other partners in the project adopted a different architectural solution - see http://ww.lpl.univ-aix.fr/projects/multext/.
} 
on SGML at the University of Edinburgh called LTNSL (Thompson and McKelvie, 1996).

The architecture is based on a commitment to TEI-style (the Text Encoding Initiative (SperbergMcQueen and Burnard, 1994)) SGML encoding of information about text. The TEI defines standard tag sets for a range of purposes including many relevant to LE systems. Tools in a LT-NSL system communicate via interfaces specified as SGML document type definitions (DTDs - essentially tag set descriptions), using character streams on pipes - an arrangement modelled after UNIX-style shell programming. To obviate the need to deal with some difficult types of SGML (e.g. minimised markup) texts are converted to a normal form before processing. A tool selects what information it requires from its input SGML stream and adds information as new SGML markup. An advantage here is a degree of data-structure independence: so long as the necessary information is present in its input, a tool can ignore changes to other markup that inhabits the same stream - unknown SGML is simply passed through unchanged (so, for example, a semantic interpretation module might examine phrase structure markup, but ignore POS tags). A disadvantage is that although graph-structured data may be expressed in SGML, doing so is complex (either via concurrent markup, the specification of multiple legal markup trees in the DTD, or by rather ugly nesting tricks to cope with overlapping - socalled "milestone tags"). Graph-structured information might be present in the output of a parser, for example, representing competing analyses of areas of text.

\subsection{Referential Approaches}

The ARPA-sponsored TIPSTER programme in the US, now entering its third phase, has also produced a data-driven architecture for NLP systems (Grishman, 1996). Whereas in LT-NSL all information about a text is encoded in SGML, which is added by the modules, in TIPSTER a text remains unchanged while information is stored in a separate database - the referential approach. Information is stored in the database in the form of annotations. Annotations associate arbitrary information (attributes), with portions of documents (identified by sets of start/end byte offsets or spans). Attributes may be the result of linguistic analysis, e.g. POS tags or textual unit type. In this way the information built up about a text by NLP modules is kept separate from the texts themselves. In place of an SGML DTD, an annotation type declaration defines the information present in annotation sets. Figure 1 shows an example from (Grishman, 1996).

\begin{tabular}{|l|l|r|r|l|}
\hline \multicolumn{5}{|c|}{$\begin{array}{c}\text { Sarah savored the soup. } \\
0 \ldots .15 \ldots\end{array}$} \\
\hline \multicolumn{4}{|c|}{ Annotations } \\
\hline \multirow{3}{*}{ Id } & Type & \multicolumn{2}{c|}{ Span } & Attributes \\
& & Start & End & \\
\hline 1 & token & 0 & 5 & pos=NP \\
2 & token & 6 & 13 & pos=VBD \\
3 & token & 14 & 17 & pos=DT \\
4 & token & 18 & 22 & pos=NN \\
5 & token & 22 & 23 & \\
\hline 6 & name & 0 & 5 & name_type=person \\
\hline 7 & sentence & 0 & 23 & \\
\hline
\end{tabular}

Figure 1: TIPSTER annotations example

The definition of annotations in TIPSTER forms part of an object-oriented model that deals with inter-textual information as well as single texts. Documents are grouped into collections, each with a database storing annotations and document attributes such as identifiers, headlines etc. The model also describes elements of information extraction (IE) and information retrieval (IR) systems relating to their use, with classes representing queries and information needs.

The TIPSTER architecture is designed to be portable to a range of operating environments, so it does not define implementation technologies. Particular implementations make their own decisions regarding issues such as parallelism, user interface, or delivery platform. Various implementations of TIPSTER systems are available, including one in GATE.

2.4 Comparison of LT-NSL and TIPSTER Both architectures are appropriate for NLP, but there are a number of significant differences. We discuss five here, then note the possibility of complimentary inter-operation of the two.

1. TIPSTER can support documents on read-only media (e.g. Internet material, or CD-ROMs, which may be used for bulk storage by organisations with large archiving needs) without copying each document.

2. From the point of view of efficiency, the original LT-NSL model of interposing SGML between all modules implies a generation and parsing overhead in each module. Later versions have replaced this model with a pre-parsed representation of SGML to reduce this overhead. This representation will presumably be stored in intermediate files, which implies an overhead from the $\mathrm{I} / \mathrm{O}$ involved in continually reading and 
writing all the data associated with a document to file. There would seem no reason why these files should not be replaced by a database implementation, however, with potential performance benefits from the ability to do I/O on subsets of information about documents (and from the high level of optimisation present in modern database technology).

3. A related issue is storage overhead. TIPSTER is minimal in this respect, as there is no inherent need to duplicate the source text and all its markup during the nromalisation process.

4. At first thought texts may appear to be onedimensional, consisting of a sequence of characters. This view breaks down when structures like tables appear - these are inherently twodimensional and their representation and manipulation is much easier in a referential model like TIPSTER than in an additive model like SGML because a markup-based representation is based on the one-dimensional view. In TIPSTER, the column of a table can be represented as a single object with multiple references to parts of the text (an annotation with multiple spans). Marking columns in SGML requires a tag for each row of the column. Related points are that: TIPSTER avoids the difficulties referred to earlier of representing graphstructured information in SGML; LT NSL is inefficient where processing algorithms require non-sequential access to data (McKelvie, Brew, and Thompson, 1997).

5. TIPSTER can easily support multi-level access control via a database's protection mechanisms - this is again not straightforward in SGML.

6. Distributed control is easy to implement in a database-centred system like TIPSTER - the DB can act as a blackboard, and implementations can take advantage of well-understood access control (locking) technology. How to do distributed control in LT-NSL is not obvious. We plan to provide this type of control in GATE via collaboration with the Corelli project at CRL, New Mexico - see (Zajac, 1997) for more details.

\subsection{Combining Addition and Reference}

We believe the above comparison demonstrates that there are significant advantages to the TIPSTER model and it is this model that we have chosen for GATE.

We also believe that SGML and the TEI must remain central to any serious text processing strategy.
The points above do not contradict this view, but indicate that SGML should not form the central representation format of every text processing system. Input from SGML text and TEI conformant output are becoming increasingly necessary for LE applications as more and more publishers adopts these standards. This does not mean, however, that flatfile SGML is an appropriate format for an architecture for LE systems. This observation is born out by the facts that TIPSTER started with an SGML architecture but rejected it in favour of the current database model, and that LT-NSL has gone partly towards this style by passing pre-parsed SGML between components.

Interestingly, a TIPSTER referential system could function as a module in an LT-NSL additive system, or vice-versa. A TIPSTER storage system could write data in SGML for processing by LT-NSL tools, and convert the SGML results back into native format. Work is underway to integrate the LT-NSL API with GATE and provide SGML I/O for TIPSTER (and we acknowledge valuable assistance from colleagues at Edinburgh in this task).

\subsection{ICE}

ICE, the Intarc Communication Environment (Amtrup, 1995), is an 'environment for the development of distributed AI systems'. As part of the Verbmobil real-time speech-to-speech translation project ICE has addressed two key problems for this type of system, viz. distributed processing and incremental interpretation (Gorz et al., 1996): distribution to contribute to processing speed in what is a very compute-intensive application area; incremental interpretation both for speed reasons and to facilitate feedback of results from downstream modules to upstream ones (e.g. to inform the selection of word interpretations from phone lattices using partof-speech information).

ICE provides a distribution and communication layer based on PVM (Parallel Virtual Machine). The infrastructure that ICE delivers doesn't fit into our tripartite classification because the communication channels do not use data structures specific to NLP needs, and because data storage and text collection management is left to the individual modules.

ICE might well form a useful backbone for an NLP infrastructure, and could operate in any of the three paradigms.

\section{NLP Trends and GATE}

For a variety of reasons NLP has recently spawned a related engineering discipline called language engineering (LE), whose orientation is towards the appli- 
cation of NLP techniques to solving large-scale, realworld language processing problems in a robust and predictable way. These problems include information extraction, text summarisation, document generation, machine translation, second language learning, amongst others. In many cases, the technologies being developed are assistive, rather than fully automatic, aiming to enhance or supplement a human's expertise rather than attempting to replace it.

The reasons for the growth of language engineering include:

- computer hardware advances which have increased processor speeds and memory capacity, while reducing prices;

- increasing availability of large-scale, languagerelated, on-line resources, such as dictionaries, thesauri, and 'designer' corpora - corpora selected for representativeness and perhaps annotated with descriptive information;

- the demand for applications in a world where electronic text has grown exponentially in volume and availability, and where electronic communications and mobility have increased the importance of multi-lingual communication;

- maturing NLP technology which is now able, for some tasks, to achieve high levels of accuracy repeatedly on real data.

Aside from the host of fundamental theoretical problems that remain to be answered in NLP, language engineering faces a variety of problems of its own. Two features of the current situation are of prime importance; they constrain how the field can develop and must be acknowledged and addressed. First, there is no theory of language which is universally accepted, and no computational model of even a part of the process of language understanding which stands uncontested. Second, building intelligent application systems, systems which model or reproduce enough human language processing capability to be useful, is a large-scale engineering effort which, given political and economic realities, must rely on the efforts of many small groups of researchers, spatially and temporally distributed, with no collaborative master plan.

The first point means that any attempt to push researchers into a theoretical or representational straight-jacket is premature, unhealthy and doomed to failure. The second means that no research team alone is likely to have the resources to build from scratch an entire state-of-the-art, LE application system. Note the tension here: the first point identifies a centrifugal tendency, pushing researchers into ever greater theoretical diversity; the second, a centripetal tendency forcing them together.

Given this state of affairs, what is the best practical support that can be given to advance the field? Clearly, the pressure to build on the efforts of others demands that LE tools or component technologies parsers, taggers, morphological analysers, discourse planning modules, etc, - be readily available for experimentation and reuse. But the pressure towards theoretical diversity means that there is no point attempting to gain agreement, in the short term, on what set of component technologies should be developed or on the informational content or syntax of representations that these components should require or produce.

Our response to these considerations has been to design and implement a software environment called GATE - a General Architecture for Text Engineering (Cunningham, Gaizauskas, and Wilks, 1995; Cunningham, Wilks, and Gaizauskas, 1996) - which attempts to meet the following objectives:

1. support information interchange between LE modules at the highest common level possible without prescribing theoretical approach (though it allows modules which share theoretical presuppositions to pass data in a mutually accepted common form);

2. support the integration of modules written in any source language, available either in source or binary form, and be available on any common platform;

3. support the evaluation and refinement of LE component modules, and of systems built from them, via a uniform, easy-to-use graphical interface which in addition offers facilities for visualising data and managing corpora.

The remainder of this paper describes the design of GATE. In section 4 we detail the design of GATE. Section 5 illustrates how GATE can be used by describing how we have taken a pre-existing information extraction system and embedded it in GATE. Section 6 makes some concluding remarks.

\section{GATE Design}

Corresponding to the three key objectives identified at the end of section 3, GATE comprises three principal elements: GDM, the GATE Document Manager, based on the TIPSTER document manager; CREOLE, a Collection of REusable Objects for Language Engineering: a set of LE modules integrated with the system; and GGI, the GATE Graphical Interface, a development tool for LE R\&D, providing 
integrated access to the services of the other components and adding visualisation and debugging tools.

Working with GATE, the researcher will from the outset reuse existing components, and the common APIs of GDM and CREOLE mean only one integration mechanism must be learnt. As CREOLE expands, more and more modules will be available from external sources (including users of other TIPSTER systems).

\subsection{GDM}

The GDM provides a central repository or server that stores all information an LE system generates about the texts it processes. All communication between the components of an LE system goes through GDM, which insulates these components from direct contact with each other and provides them with a uniform API for manipulating the data they produce and consume.

The basic concepts of the data model underlying the GDM have been explained in the discussion of the Tipster model in section 2.3 above. The TIPSTER architecture has been fully specified (Grishman, 1996) and its specification should be consulted for further details, in particular for definitions of the API. The GDM is fully conformant with the core document management subset of this specification.

\subsection{CREOLE}

All the real work of analysing texts in a GATE-based LE system is done by CREOLE modules or objects (we use the terms module and object rather loosely to mean interfaces to resources which may be predominantly algorithmic or predominantly data, or a mixture of both). Typically, a CREOLE object will be a wrapper around a pre-existing LE module or database - a tagger or parser, a lexicon or ngram index, for example. Alternatively, objects may be developed from scratch for the architecture - in either case the object provides a standardised API to the underlying resources which allows access via GGI and I/O via GDM. The CREOLE APIs may also be used for programming new objects.

When the user initiates a particular CREOLE object via GGI (or when a programmer does the same via the GATE API when building an LE application) the object is run, obtaining the information it needs (document source, annotations from other objects) via calls to the GDM API. Its results are then stored in the GDM database and become available for examination via GGI or to be the input to other CREOLE objects.

GDM imposes constraints on the I/O format of CREOLE objects, namely that all information must be associated with byte offsets and conform to the annotations model of the TIPSTER architecture. The principal overhead in integrating a module with GATE is making the components use byte offsets, if they do not already do so.

\subsection{GGI}

The GGI is a graphical tool that encapsulates the GDM and CREOLE resources in a fashion suitable for interactive building and testing of LE components and systems. The GGI has functions for creating, viewing and editing the collections of documents which are managed by the GDM and that form the corpora which LE modules and systems in GATE use as input data. The GGI also has facilities to display the results of module or system execution new or changed annotations associated with the document. These annotations can be viewed either in raw form, using a generic annotation viewer, or in an annotation-specific way, if special annotation viewers are available. For example, named entity annotations which identify and classify proper names (e.g. organization names, person names, location names) are shown by colour-coded highlighting of relevant words; phrase structure annotations are shown by graphical presentation of parse trees. Note that the viewers are general for particular types of annotation, so, for example, the same procedure is used for any POS tag set, Named-Entity markup etc. (see section 4.4 below). Thus CREOLE developers reuse GATE data visualisation code with negligible overhead.

\subsection{Plug and Play}

The process of integrating existing modules into GATE (CREOLEising) has been automated to a large degree and can be driven from the interface. The developer is required to produce some $\mathrm{C}$ or Tcl code that uses the GDM TIPSTER API to get information from the database and write back results. When the module pre-dates integration, this is called a wrapper as it encapsulates the module in a standard form that GATE expects. When modules are developed specifically for GATE they can embed TIPSTER calls throughout their code and dispense with the wrapper intermediary. The underlying module can be an external executable written in any language (the current CREOLE set includes Prolog, Lisp and Perl programs, for example).

There are three ways to provide the CREOLE wrapper functions. Packages written in $\mathrm{C}$, or in languages which obey $\mathrm{C}$ linkage conventions, can be compiled into GATE directly as a Tcl package. This is tight coupling and is maximally efficient but necessitates recompilation of GATE when modules change. On platforms which support shared 
libraries C-based wrappers can be loaded at runtime - dynamic coupling. This is also efficient (with a small penalty at load time) and allows developers to change CREOLE objects and run them within GATE without recompiling the GATE system. Wrappers written in Tcl can also be loaded at run-time - loose coupling. There is a performance penalty in comparison with using the $\mathrm{C}$ APIs, but for simple cases this is the easiest integration route. In each case the implementation of CREOLE services is completely transparent to GATE.

CREOLE wrappers encapsulate information about the preconditions for a module to run (data that must be present in the GDM database) and post-conditions (data that will result). This information is needed by GGI, and is provided by the developer in a configuration file, which also details what sort of viewer to use for the module's results and any parameters that need passing to the module. These parameters can be changed from the interface at run-time, e.g. to tell a parser to use a different lexicon. Aside from the information needed for GGI to provide access to a module, GATE compatibility equals TIPSTER compatibility - i.e. there will be very little overhead in making any TIPSTER module run in GATE.

Given an integrated module, all other interface functions happen automatically. For example, the module will appear in a graph of all modules available, with permissible links to other modules automatically displayed, having been derived from the module pre- and post-conditions.

At any point the developer can create a new graph from a subset of available CREOLE modules to perform a task of specific interest.

\section{VIE: An Application In GATE}

To illustrate the process of converting pre-existing LE systems into GATE-compatible CREOLE sets we use as an example the creation of VIE (Vanilla Information Extraction system) from LaSIE (LargeScale Information Extraction system) (Gaizauskas et al., 1995), Sheffield's entry in the MUC-6 system evaluations. LaSIE module interfaces were not standardised when originally produced and its CREOLEization gives a good indication of the ease of integrating other LE tools into GATE. The resulting system, VIE, is distributed with GATE.

\subsection{LaSIE}

LaSIE was designed as a research system for investigating approaches to information extraction and to be entered into the MUC-6 conference (Grishman and Sundheim, 1996). As such it was a stand- alone system that was aimed at specific tasks and, while based on a modular design, none of its modules were specifically designed with reuse in mind, nor was there any attempt to standardise data formats passed between modules. Modules were written in a variety of programming languages, including $\mathrm{C}, \mathrm{C}++$, Flex, Perl and Prolog. In this regard LaSIE was probably typical of existing LE systems and modules. The high-level tasks which LaSIE performed include the four MUC-6 tasks (carried out on Wall Street Journal articles) - named entity recognition, coreference resolution and two template filling tasks. The system was a pipelined architecture which processes a text sentence-at-a-time and consists of three principal processing stages: lexical preprocessing, parsing plus semantic interpretation, and discourse interpretation.

\subsection{The CREOLEisation of LaSIE}

As described in section 4.2, CREOLEisation of existing LE modules involves providing them with a wrapper so that the modules communicate via the GDM, by accessing TIPSTER-compliant document annotations and updating them with new information. The major work in converting LaSIE to VIE involved defining useful module boundaries, unpicking the connections between them, and then writing wrappers to convert module output into annotations relating to text spans and to convert GDM input from annotations relating to text spans back into the module's native input format.

The complete VIE system comprises ten modules, each of which is a CREOLE object integrated into GATE. The CREOLEisation took approximately two person months. The resulting system has all the functionality of the original LaSIE system. However, the interface makes it much easier to use. And, of course, it is now possible to swap in modules, such as a different parser, with significantly less effort than would have been the case before. For more details of this process see (Cunningham et al., 1996).

VIE and its components are being deployed for a number of purposes including IE in French, German and Spanish. Experience so far indicates that GATE is a productive environment for distributed collaborative reuse-based software development.

\section{Concluding Remarks}

Of course, GATE does not solve all the problems involved in plugging diverse LE modules together. There are three barriers to such integration:

- managing storage and exchange of information about texts; 
- incompatibility of representation of information about texts;

- incompatibility of type of information used and produced by different modules.

GATE provides a solution to the first two of these, based on the work of the TIPSTER architecture group. Because GATE places no constraints on the linguistic formalisms or information content used by CREOLE modules, the latter problem must be solved by dedicated translation functions - e.g. tagset-to-tagset mapping - and, in some cases, by extra processing - e.g. adding a semantic processor to complement a bracketing parser.

The recent completion of this work means a full assessment of the strengths and weaknesses of GATE is not yet possible. The implementation of VIE in GATE, however, provides an existence proof that the original conception is workable. We believe that the environment provided by GATE will now allow us to make significant strides in assessing alternative LE technologies and in rapidly assembling LE prototype systems. Thus, to return to the themes of section 3, GATE will not commit us to a particular linguistic theory or formalism, but it will enable us, and anyone who wishes to make use of it, to build, in a pragmatic way, on the diverse efforts of others.

\section{Acknowledgements}

This work was supported by the UK Engineering and Physical Sciences Research Council, grant number GR/K25267, and the EC DG XIII Language Engineering programme, grant number LE1-2238.

\section{References}

Amtrup, J.W. 1995. ICE - INTARC Communication Environment User Guide and Reference Manual Version 1.4. Technical report, University of Hamburg.

Cunningham, H., R.G. Gaizauskas, and Y. Wilks. 1995. A General Architecture for Text Engineering (GATE) - a new approach to Language Engineering R\&D. Technical Report CS - 95 - 21, Department of Computer Science, University of Sheffield. Also available as http://xxx.lanl.gov/ps/cmp-lg/9601009.

Cunningham, H., K. Humphreys, R. Gaizauskas, and M. Stower, 1996. CREOLE Developer's Manual. Department of Computer Science, University of Sheffield. Available at http://www.dcs.shef .ac.uk/research/groups/ nlp/gate.
Cunningham, H., Y. Wilks, and R. Gaizauskas. 1996. GATE - a General Architecture for Text Engineering. In Proceedings of the 16th Conference on Computational Linguistics (COLING96), Copenhagen, August.

Gaizauskas, R., T. Wakao, K Humphreys, H. Cunningham, and Y. Wilks. 1995. Description of the LaSIE system as used for MUC-6. In Proceedings of the Sixth Message Understanding Conference (MUC-6). Morgan Kaufmann.

Gorz, G., M. Kessler, J. Spilker, and H. Weber. 1996. Research on Architectures for Integrated Speech/Language Systems in Verbmobil. In Proceedings of COLING-96, Copenhagen.

Grishman, R. 1996. TIPSTER Architecture Design Document Version 2.2. Technical report, DARPA. Available at http://www.tipster.org/.

Grishman, R. and B. Sundheim. 1996. Message understanding conference - 6: A brief history. In Proceedings of the 16th International Conference on Computational Linguistics, Copenhagen, June.

McKelvie, D., C. Brew, and H. Thompson. 1997. Using SGML as a Basis for Data-Intensive NLP. In Proceedings of the fifth Conference on Applied Natural Language Processing (ANLP-97).

Schütz, J. 1994. Developing Lingware in ALEP. ALEP User Group News, CEC Luxemburg, 1(1), October.

Shieber, S. 1992. Constraint-Based Grammar Formalisms. MIT Press.

Simkins, N. K. 1994. An Open Architecture for Language Engineering. In First Language Engineering Convention, Paris.

Sperberg-McQueen, C.M. and L. Burnard. 1994. Guidelines for Electronic Text Encoding and Interchange (TEI P3). ACH, ACL, ALLC.

Thompson, H.S. and D. McKelvie. 1996. A Software Architecture for Simple, Efficient SGML Applications. In Proceedings of SGML Europe '96, Munich.

Zajac, R. 1997. An Open Distributed Architecture for Reuse and Integration of Heterogenous NLP Components. In Proceedings of the 5th conference on Applied Natural Language Processing (ANLP97). 